ISSN No. 0974-035X

An Indexed, Refereed \& Peer Reviewed Journal of Higher Education

Towards Excellence

UGC-HUMAN RESOURCE DEVELOPMENT CENTRE,

GUJARAT UNIVERSITY, AHMEDABAD, INDIA

\title{
ડુંગરના ખોળે
}

sૉ. aी. કे. ચાasા

વર્ષોથી થલતેજની વસંત નેચર ક્યોર હેસ્પિટલમાં જાતે બનાવેલ વોલીબોલના કોર્ટ ઉપર વહેલી સવારે નિયમિત વોલીબોલ રમવાની માનસિકતાએ મજબુત પકડ જમાવેલ છે. ઓફિસ સમય બાદ ફુરસદના સમટે વસંત નેચર ક્યોર હોસ્પિટલમાં બેસવાની વર્ષોની ટેવ હતી. બગીચાના હીંચકે બેસી ગુજરાતી સુગમ સંગીત, ભજનો સાંભળવાનો શીખ કાયમ હતો. 17 જૂન, 2016 નો દિવસ હતી, આ દિવસે મારો જન્મ દિવસ હતો. હિંચકે બેસી સાંજના સમટે ભજન સાંભળતો હતો. મનમાં વિચાર આવ્યો આજે પચ્યાસ વર્ષ પૂર્ણ થયા. આવતી કાલથી એકાવનમાં વર્ષમાં પ્રવેશ થશે. જેન ગુજરાતી કહેવત મુજબ "વનપ્રવેશ" કહે છે. મનમાં પચ્યાસ વર્ષની ઉજવણી અનોખી રીત કરવાની ઇચ્છા થઈ. મનમાં ગડમથલ ચાલતી હતી કે એવું કશુંક કરવું જે જીવનભરનું સંભારણું બને તથા ભાવી પેઢીને પ્રેરણાદાયી બને.

ધરતીપુત્ર હોવાને કારણે પ્રથમથી જ શારીરિક પ્રવૃત્તિઓ, ધોડેસવારી તથા કુદરતના ખોળે રમવાની ભમણ પ્રવૃત્તિ પ્રત્ટે સહજ આકર્ષણ રહેલ. મારા શોખન પોષી શકે તેવી યુવાનોની પ્રવૃત્તિ કરવાની તક ગુજરાત યુનિવર્સિટીમાં નોકરીમાં જોડાઈને મળી. વર્ષોથી યુનિવર્સિટીના વિદ્યાર્થીઓ માટે ટ્રેકિંગ, માઉન્ટેનિયરીંગ જેવી પ્રવૃત્તિઓ ગુજરાતના અલગ-અલગ જંગલો, દરિયા કિનારે, રણ તથા પહાડોમાં કરાવતો હતો. તેથી તે દિશામાં વિચારોનું મનીમંથન ચાલ્યું, મનમાં વિચાર સ્ક્ર્યો, ગુજરાતમાં ધણા પર્વતી આવેલા છે. તે પર્વતીનું ચઢાણ કરુ તો કેવું રહે. ગુજરાતના ખ્ચાતનામ પર્વતીનું ચઢાણ એકાવનમાં વર્ષના એક વર્ષના સમયગાળા દરમિયાન કરવું. આ વિચાર મેં મારી પત્ની તેમજ પુત્ર સમક્ષ મુક્યો અને તેઓએ મારી આ લાગણીને સહષ્ષ વધ્ધાવી લીધી અને એને પૂણ્ણ કરવા પ્રોर્યो. મેં સંકલ્પ કર્યો વનપ્રવેશના એકાવનમાં એક વર્ષના સમયગાળામાં ગુજરાતના ખ્યાતનામ પર્વતીનું ચઢાણ કરી, યુવાનોને આ દિશામાં વિચારવાની તક પુરી પાડી તેમનામાં સાહસિક પ્રવૃત્તિઓ પ્રત્ટ આકર્ષણ થાય અને સાથે સાથે ગુજરાતનું યુવાધન સાહસિક બની એડવેન્યર એકટીવીટીમાં જોડાય.

મારા આ સંકલ્પ વિષે જણાવું તો મેં ગુજરાતના 21 પર્વતોનું ચઢાણ કર્યું છે (આ પર્વતીની વિશેષ માહિતી આ સાથે સામેલ છે). તેમાંથી કેટલાક ખ્ચાતનામ છે જ્યારે કેટલાક ઓછા જાણીતા પર્વતો છે. છતાં પણ દરેક પર્વતનું નિજીપણું તો છે જ. ગિરનાર પર્વત ગુજરાતના પર્વતીમાં સૌથી વધું ઉંચાઈ ધરાવતી 
Towards Excellence: An Indexed, Refereed \& Peer Reviewed Journal of Higher Education / Dr. V.K. Chavda / Page 99-102

હિમાલય કરતા પણ જૂનો પર્વત છે. ગબ્બર, ઈડરનો ડુંગર, તારંગા, ગિરનાર, ઓસમ, દાતાર, ચોટીલા, ભુજ્યો ડુંગર, ઘિણોધર, શેત્રુંજય જેવા પર્વતો પર સીધા જ પગથીયાથી ચઢાણ કરી શકાય છે. ડોશીમાંનો ભાખરો, જેસોર, બરડો (આભાપરા), નવનાથધુણા, કનડા ડુંગર, પાવાગઢ જેવા પર્વતો પર કેડીઓના રસ્તે પગપાળા ટ્રેકિંગ કરી ચઢાણ કરી શકાય છે. કાળો ડુંગર, રતનમહાલ, સાપુતારા, ડોન, વિલ્સન હિલ પર્વતો પર મોટર માર્ગે છેક ઉપર સુધી જઈ શકાય છે. સૌથી અઘરું અને કપરું ચઢાણ નવનાથધુણાના પર્વતનું છે. કેટલુંક ચઢવાનું તથા ઉતરવાનું ફક્ત બેસીને જ ફરજિયાત પણે કરવું પડે તેવું વિકટ છે. રોક ક્લાઈમ્બિંગની શ્રેષ્ઠ સાઈટી આ પર્વત પર જોવા મળે છે. તફઉપરાંત ગિરનાર, દાતાર, જેસોર જેવા પર્વતોમાં પણ નાની મોટી ક્લિફેમાં રોક ક્લાઈમ્બિંગ કરવાની ઉત્તમ તકો રહેલી છે. નવનાથધુણા, ગિરનાર, દાતાર, જેસોર, ડોશીમાનો ભાખરો, પાવાગઢ જેવા પર્વતોમાં માઉન્ટેનિયરીંગ માટેની પ્રવૃત્તિઓ થઈ શકે તેવી ઘણી બધી સાઈટો જોવા મળે છે. મોટાભાગના બધા જ પર્વતો પર ટ્રેકિંગની પ્રવૃત્તિઓ થઈ શકે તેમ છે. સૌંદર્યથી ભરપૂર અને કુદરતના અફાટ ખજાના સમા જેસોર પર્વતમાં મને સૌથી વધારે આનંદ અને પરમ સુખની અનુભૂતી થઈ. જેસોર પર્વતના ચઢાણ દરમિયાન રસ્તામાં મોટાં મોટાં વડલાના વૃક્ષો મોટી સંખ્યામાં જોવા મળ્યા, જે બીજા પર્વતો પર ક્યાંય જોવા મળતા નથી. જેસોર તથા રતનમહાલ પર્વતોના વિસ્તારમાં રીંછ અભ્યારણ્ય આવેલ છે. કાળા ડુંગરની અનુભૂતિ પણ અદ્વિતીય છ. પર્વતની ટોચ પરથી ઔક તરફ અફાટ રણની નજારી તથા બીજી તરફ ધુધવાતા સમુદ્રના મોજા નિહાળવાની અવિસ્મરણીય ક્ષણો હંમેશ માટે યાદગાર બની રહેશ. કચ્છની સરહદ નજીક હોવાથી કાળા ડુંગર પર BSF ની ચોકી પણ જોવા મળે છે. કનડા ડુંગર ઐતિહાસિક ધરોહર ધરાવતો ડુંગર છે. જ્યાં 1882 માં જુનાગઢ નવાબની ફોજ દ્વારા દગાફટકાથી ગોળીબારમાં શહિદ થનાર 82 મહિયા રાજપૂતોની ખાંભીઓનું શહિદી સ્મારક ડુંગરની ટોચ ઉપર આવેલું છે. તફઉપરાંત જેસલ અને જખરા (ઓઢાજામ-હોથલના પુત્રો) જેવા વીરપુરૂષોનું જન્મસ્થળ જ્યાં બાળપણમાં બંને ભાઈઓ સિંહના બચ્યા સાથે રમતા હતા. સાપુતારા પર્વત પર ગુજરાતનું એક માત્ર હિલ સ્ટેશન આવેલ છે. ડોન પર્વત પર પણ હિલ સ્ટેશન બની શકે તેવી ભૌગોલિક પરિસ્થિતી જોવા મળે છે. ડોન પર્વતને હિલ સ્ટેશન તરીકે વિકસાવવાની તાતી જરૂર છે.

ઉત્તર ગુજરાતના પર્વતો અરવલ્લી રેન્જના ભાગરૂપે જોવા મળે છે. જ્યારે સૌરાષ્ટ્રના પર્વતો માંડવ હિલની ટેકરીઓના ભાગરૂપે જોવા મળે છે. કચ્છ પ્રદેશના પર્વતો દક્ષિણ ભારતના દ્વિકલ્પીય પ્રદેશના ખડક સંરચનાના ભાગરૂપે જોવા મળે છે. પાવાગઢ તથા રતનમહલ જેવા પર્વતો વિધ્યાંચલની પર્વત શૃંખલાઓમાં આવેલ છે. જ્યારે પશ્વિમ ઘાટના સહ્યાદ્રીના ભાગરૂપે દક્ષિણ ગુજરાતના પર્વતીય ક્ષેત્રોનો સમાવેશ થાય છે. 
Towards Excellence: An Indexed, Refereed \& Peer Reviewed Journal of Higher Education / Dr. V.K.

ગુજરાતના મોટા ભાગના પર્વતો ઉપર લોકોની આસ્થાના પ્રતિક સમા દેવ-દેવીઓના નાના-મોટા મંદિરો, આશ્રમો, સ્થાનકો જોવા મળે છે. લોકો ધાર્મિક લાગણીઓથી જોડાઈને આવા પર્વતો પર ચઢાણ કરતા જોવા મળે છે. ધાર્મિક આસ્થાના કારણે જ આવા પર્વતો પર નાના બાળકોથી માંડીને મોટી ઉંમરના વૃદ્ધો પણ ચઢાણ કરતા જોવા મળ્યા, તેમાંય ખાસ કરીને આરાસુર (ગબ્બર) અને પાવાગઢ પર્વત પર શ્રદ્ધાળુઓની વિશેષ હાજરી જોવા મળી. આ બંને પર્વતો પર પર્વતોની ટોચ પર પહોંચવા માટે રોપ-વેની વ્યવસ્થા હોવાને કારણે પ્રવાસીઓની સંખ્યા વિશેષ જોવા મળે છે. લોકમેળો ભરાયો હોય તેવો અહેસાસ થાય છે અને તેના કારણે જ આ બંને પર્વતો પર કચરાના ઢગલા તથા ગંદકી જોવા મળે છે. આવા રમણીય પર્વતોની સુંદરતા હણાતી જોઈ આઘાત સાથે દુ:ખની લાગણી થઈ. સ્વચ્છતાની દ્રષ્ટીઓ શેત્રુંજય પર્વત શ્રેષ્ઠ છે. ક્યાંય પ્લાસ્ટિક, ગંદકી કે કચરાના ઢગલા જોવા મળતા નથી. સ્વચ્છતાનું આ ભગીરથ કાર્ય શેઠ આણંદજી કલ્યાણજી પેઢી દ્વારા કરવામાં આવે છે, તેમ જાણવા મળ્યું.

ગુજરાતના યુવાનો તથા નાના-મોટા સૌને રમણીય સ્થળોએ ફરવા જવાનો શોખ સૌ જાણે છે. સમગ ભારતમાંથી સૌથી વધારે પ્રવાસની મજા માણતા હોય તી તે ગુજરાતીઓ છે. પરંતુ આપણા ગુજરાતમાં જ આવા સ્થળોની અવકાશ ખૂબ જ જૂજ જોવા મળી રહ્યો છે! ગુજરાતીઓને એડવેન્યર એક્ટીવીટીનું ખૂબ જ આકર્ષણ રહેલું છે. પરંતુ ગુજરાતમાં તેની તકો ખૂબ જ મર્યાદિત જોવા મળી રહી છે અને માટે જ લોકોનું ધાર્મિક સ્થળોએ પ્રવાસે જવાનું પ્રયોજન વિશેષ જોવા મળી રહ્યું છે, તેવું મારું માનવું $\xi$ छे.

ગુજરાતના ખોળે આવા ખૂબજ રમણીય પર્વતો કુદરતે આપ્યા છે ત્યારે આવા કુદરતના અપાર સૌંદર્ય ધરાવતા પર્વતોને પર્યટન સ્થળો તરીકે વિકસાવવા જોઈએ. સાથે સાથે દરેક પર્વતી પર માઉન્ટેનિયરીંગ, ટ્રેકિંગ, પ્રાકૃતિક શિક્ષણ તથા ઈકો એડવેન્યર સ્પોર્ટસ એક્ટિવિટીનો વિકાસ થઈ શકે તેવી વિશેષ સગવડો ઊભી કરી કેમ્પ સાઈટો ઊભી કરવી જોઈએ. જેથી ખાસ કરીને ગુજરાતનું યુવાધન સાહસિક પ્રવૃત્તિમાં જોડાઈ પોતાની સાહસિક વૃત્તિને પોષી શકે. યુવાનો સાથેના સતત સહવાસને કારણે યુવાનોની નિષ્રિયતા તથા દિશા વિહિનતાથી ખૂબ જ ચિંતિત છું. યુવાધન સમાજની કરોડરજજજ છે. તેમનામાં શક્તિઓનો અખૂટ પ્રવાહ વહેતો રહે છે. તેઓ તે શક્તિઓનો ઉપયોગ કરવાનું જાણે છે, પરંતુ જ્યાં સુધી તેમનામાં આત્મવિશ્ચાસ જાગૃત નહીં થાય ત્યાં સુધી તેઓ પોતાની શક્તિને ઓળખીને તેની સદ્દપયોગ નહીં કરી શકે અને તેથી જ આ ઉંમરને શાલિન બનાવવા માટે શિક્ષણમાં વ્યાયામ, સ્વાવલંબન, કલા, કૌશલ અને સ્વાધ્યાય સાથે સાથે માઉન્ટેનિયરીંગ તથા ટ્રેકિંગ જેવી પ્રવૃત્તિઓનો સમાવેશ કરવો જોઈએ એવું આટલા વર્ષોના અનુભવ પરથી હું દ્રઢ પણે માનું છું. ગુજરાતનો યુવાન આવી પ્રવૃત્તિમાં જોડાવા થનગને છે તેન જરૂર છે ફક્ત તક પુરી પાડવાની. આ તક પુરી પાડવાની જવાબદારી શિક્ષણ જગત સાથે સંકળાયેલા 
Towards Excellence: An Indexed, Refereed \& Peer Reviewed Journal of Higher Education / Dr. V.K.

આપણા સૌની છે. આ તક પુરી પાડવાનું ભગીરથ કાર્ય હાલમાં ગુજરાત યુનિવર્સિટી દ્વારા યુવાધન માટે જંગલ, દરિયા કિનારે, રણ તથા પર્વતોમાં વિવિધ કેમ્પોનું આયોજન કરીને કરવામાં આવી રહ્હું છે, તેન આનંદ સહ આવકારું છું. આ માટે ગુજરાત યુનિવર્સિટીના સત્તાધિશોને અભિનંદન પાઠવું છું તથા કૃતજ્ઞ ભાવે તેમનો આભાર વ્યક્ત કરું છું.

સમયની અનુકુળતા અને ફુરસદ મળે તો મારી આ પર્વતારોહણની સફર દરમ્યાન થયેલ અનુભૂતિ, પર્વતોની સુંદરતા, પર્વતોની સંરચના, પર્વતો પર આવેલ ધાર્મિક સ્થળો, તેમાં રહેલા જંગલો, વનસ્પતિ, પ્રાણીઓ, ચઢાણના રસ્તાઓ તેમજ આ તમામ પર્વતોનું સાંસ્કૃતિક અન ઐતિહાસિક મૂલ્ય વિગેરેની વિસ્તૃત છણાવટ "ગુજરાતના પર્વતો” નામે પુસ્તિકા સ્વરૂપે પ્રગટ કરવાની નેમ છે. સાથે સાથે કુદરત સાથ આપે અને શારીરિક તંદુરસ્તી સારી રહે તો એકાવનમાં વર્ષ જે પર્વતોનું ચઢાણ કર્યું છે તે બધા જ પર્વતોનું ચઢાણ દશ વર્ષ પછી એટલે કે એકસઠમાં વર્ષે કરવાની અંતરની ઇચ્છા છે અને છેલ્લે એટલું જ કહીશ...

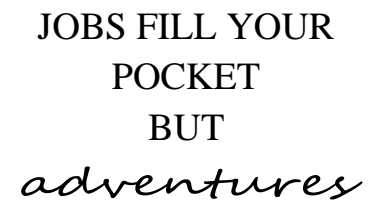

FILL YOUR

SOUL

\author{
sૉ. वी. કे. ચાવsા \\ મદદનીશ નિયામક, યુવક કલ્યાણ \\ ગુજરાત યુનિવર્સિટી, અમદાવાદ
}

